\title{
Hipertireoidismo felino secundário à adenocarcinoma de tireóide
}

\author{
Feline hyperthroidism secondary to thyroid adenocarcinoma \\ Hipertiroidismo felino secundario al adenocarcinoma tiroideo
}

Recebido: 29/11/2021 | Revisado: 07/12/2021 | Aceito: 11/12/2021 | Publicado: 19/12/2021

Renata Borges da Silva

ORCID: https://orcid.org/0000-0001-8246-747X

Universidade Estadual do Ceará, Brasil

E-mail: renataelborges@gmail.com

Vitor Lima Torres

ORCID: https://orcid.org/0000-0002-5806-6905 Universidade Estadual do Ceará, Brasil E-mail: vitortorres.mv@outlook.com

Amanda Brena Freitas Ribeiro

ORCID: https://orcid.org/0000-0002-6872-326X Universidade Estadual do Ceará, Brasil E-mail: amandabrenafr@gmail.com

Reginaldo Pereira de Sousa Filho

ORCID: https://orcid.org/0000-0002-3643-4375 Universidade Estadual do Ceará, Brasil E-mail: reginaldo.catus@gmail.com

Francisco Douglas Lima Anastácio ORCID: https://orcid.org/0000-0001-6943-4796 Universidade Estadual do Ceará, Brasil E-mail: douglasanastacio7@gmail.com

Léia Jamylle dos Santos Freitas ORCID: https://orcid.org/0000-0002-4183-447X Universidade Estadual do Ceará, Brasil E-mail: leiajamyllefreitas@gmail.com

Glenda Roberta Freire Lima

ORCID: https://orcid.org/0000-0002-3867-7389 Universidade Estadual do Ceará, Brasil

E-mail: glendaroberta.medvet@gmail.com

Letícia Caetano Siqueira

ORCID: https://orcid.org/0000-0001-5826-6459

Universidade Federal Rural do Semi-Árido, Brasil E-mail: leticiacaaetano@ hotmail.com

Germano Gonçalves Teixeira

ORCID: https://orcid.org/0000-0002-6586-1819 Universidade Estadual do Ceará, Brasil

E-mail: germanoggt06@gmail.com

\begin{abstract}
Resumo
O hipertireoidismo refere-se ao aumento dos hormônios tireóideos funcionais, tiroxina (T4) e tri-iodotironina (T3). A maioria dos casos de hipertireoidismo é causada pelo adenoma tireóideo ou pela hiperplasia adenomatosa multinodular, afetando um ou ambos os lobos da glândula tireóide. Menos de 5\% dos casos ocorrem como resultado de carcinoma tireóideo. Dessa forma, este trabalho teve como objetivo apresentar um relato de caso acerca do hipertireoidismo felino ocasionado por carcinoma na tireoide, em um felino macho de 11 anos. O animal apresentou histórico de anorexia, vômito e diarréia há cerca de um mês. Foram solicitados hemograma completo, bioquímico, ultrassonografia abdominal e tomografia da região cervical. Os exames laboratoriais e físico indicaram fortes indícios de hipertireoidismo, como eritrocitose, aumento de T4 total e aumento do volume palpável da tireóide, além de exame tomográfico indicativo de processo neoplásico em lobo tireoidiano esquerdo, sem acometimento de estruturas adjacentes. $\mathrm{O}$ tratamento de escolha foi o cirúrgico com a técnica extracapsular modificada com abordagem cervical (tireodectomia com preservação das glândulas paratireoides). Após a realização dos procedimentos cirúrgicos e tratamento de suporte o animal apresentou remissão dos sinais clínicos.
\end{abstract}

Palavras-chave: Endocrinopatia; Adenocarcinoma; Felino.

\footnotetext{
Abstract

Hyperthyroidism refers to the increase in functional thyroid hormones, thyroxine (T4) and triiodothyronine (T3). Most cases of hyperthyroidism are caused by thyroid adenoma or multinodular adenomatous hyperplasia, affecting one or both lobes of the thyroid gland. Less than $5 \%$ of cases occur as a result of thyroid carcinoma. Thus, this study aimed to
} 
present a case report about feline hyperthyroidism caused by thyroid carcinoma, in an 11-year-old male feline. The animal had a history of anorexia, vomiting and diarrhea about a month ago. Complete blood count, biochemical, abdominal ultrasound and CT scan of the cervical region were requested. Laboratory and physical examinations indicated strong signs of hyperthyroidism, such as erythrocytosis, increase in total T4 and increase in the palpable volume of the thyroid, in addition to tomographic examination indicative of neoplastic process in the left thyroid lobe, without involvement of adjacent structures. The treatment of choice was surgery with the modified extracapsular technique with a cervical approach (thyrodectomy with preservation of the parathyroid glands). After surgical procedures and supportive treatment, the animal presented remission of clinical signs.

Keywords: Endocrinopathy; Adenocarcinoma; Feline.

\section{Resumen}

El hipertiroidismo se refiere al aumento de las hormonas tiroideas funcionales, la tiroxina (T4) y la tri-yodotironina (T3). La mayoría de los casos de hipertiroidismo son causados por adenoma tiroideo o hiperplasia adenomatosa multinodular, que afecta a uno o ambos lóbulos de la glándula tiroides. Menos del 5\% de los casos ocurren como resultado de carcinoma de tiroides. Así, este estudio apuntó presentar un informe del caso sobre el hipertiroidismo felino causado por carcinoma de la tiroides en un felino masculino de 11 años. El animal tenía antecedentes de anorexia, vómitos y diarrea durante aproximadamente un mes. El ultrasonido completo, bioquímico, abdominal y la tomografía de la región cervical fueron solicitados. El laboratorio y los pruebas físicos indicaron indicaciones fuertes del hipertiroidismo, tales como erythrolithosis, T4 total creciente y volumen palpable creciente de la tiroides, además de la examinación tomográfica indicativa de proceso neoplástico en lóbulo izquierdo de la tiroides, sin la implicación de estructuras adyacentes. El tratamiento de la opción era quirúrgico con la técnica extracapsular modificada con el acercamiento cervical (thyrodectomy con la preservación de las glándulas paratiroides). Después de realizar los procedimientos quirúrgicos y el tratamiento de apoyo, el animal presentó remisión de los signos clínicos.

Palabras clave: Endocrinopatía; Adenocarcinoma; Felino.

\section{Introdução}

O hipertireoidismo é uma endocrinopatia causada pela excessiva produção e secreção dos hormônios tiroxina (T4) e triiodotironina (T3) pela glândula tireóide anormal, ocorre com maior frequência em gatos de idade avançada e sem raça definida (Peterson, 2012). Os sinais clínicos resultantes variam, porém quase sempre são hiperatividade, polifagia, perda de peso, poliúria, polidipsia, diarréia e taquicardia. No exame físico, geralmente é possível observar pelagem opaca, má condição clínica e aumento da glândula tireóide (Guun-Moore, 2005).

A maioria dos casos de hipertireoidismo felino, são ocasionados por hiperplasia adenomatosa envolvendo um lobo da tireóide ou ambos, de caráter benigno. O carcinoma de tireóide em gatos é um distúrbio incomum, com uma prevalência de menos de 5\% de todos os casos de hipertireoidismo felino. (Vail et al., 2019). Dessa forma, este trabalho teve como objetivo apresentar um relato de caso acerca do hipertireoidismo felino ocasionado por carcinoma de tireoide, em um felino macho de 11 anos.

\section{Metodologia}

Foi atendido em uma clínica veterinária particular na cidade de Fortaleza/CE, um felino, macho, sem raça definida, de 11 anos de idade, castrado e com vermifugação e vacinação atualizadas, com histórico de anorexia, vômito e diarréia há cerca de um mês e com um recente caso de bronquite. No exame clínico foi observado aumento da tireóide a palpação, conforme a técnica de Norsworthy et al., (2002), perda de massa muscular e taquicardia (240 bpm). Foram solicitados exames hematológicos, bioquímicos, ultrassonográficos, tomografia de região cervical e após procedimento cirúrgico, o histopatológico. O tratamento inicial instituído consistiu em metimazol, porém, posteriormente foram necessárias duas tireoidectomias, com intervalo de 30 dias. 


\section{Resultados e Discussão}

No hemograma inicial, foi verificado aumento das enzimas hepáticas ALT (267 UI/L), AST (73 UI/L) e FA (155 UI/L). Isto pode ter ocorrido provavelmente pela má nutrição, anóxia hepática e do efeito tóxico direto dos hormônios tireoidianos no fígado (Daleck \& De Nardi, 2016). Além disso, no mesmo período também foi observada discreta eritrocitose (10,28 M/uL), o que também é recorrente em animais com hipertireoidismo, e que pode ser explicado pelo elevado consumo de oxigênio e pelo estímulo ß-adrenérgico sobre a medula óssea, elevando a eritropoese (Mooney, 2001). Os valores observados no hemograma do paciente estão esquematizados na Tabela 1.

Tabela 1: tabela comparativa dos valores observados no hemograma do paciente ao longo de todo tratamento.

\begin{tabular}{|c|c|c|c|c|c|c|}
\hline & \multicolumn{2}{|c|}{ Antes } & \multicolumn{2}{|c|}{$25 / 06-1^{a}$ cirurgia } & \multicolumn{2}{|c|}{$05 / 08-2^{\mathrm{a}}$ cirurgia } \\
\hline Data & $02 / 05$ & $22 / 05$ & $19 / 06$ & $05 / 08$ & $18 / 08$ & 08/09 \\
\hline Hemácias & 10,28 & 8,48 & 8,91 & 4,8 & 5,7 & 8,5 \\
\hline Hematócrito & 45 & 34,9 & 33,9 & 20,7 & 25,6 & 33,5 \\
\hline Hemoglobia & 16,8 & 13,3 & 13,9 & 7,6 & 10,5 & 11,6 \\
\hline$V G M$ & 16,4 & 41,2 & 38,1 & 42,6 & 44,7 & 39,1 \\
\hline CHCM & 37,4 & 38 & - & 37 & & 34,5 \\
\hline Plaquetas & 179000 & 86000 & 49000 & 101000 & 210000 & 109000 \\
\hline
\end{tabular}

Fonte: Autores.

Os achados tomográficos indicaram presença de massa localizada em região cervical ventral com aspecto homogêneo em seu interior, contornos regulares e limites indefinidos em fase pré-contraste, após a administração do contraste iodado intravenoso observou-se aspecto bilobado da massa, com sua porção caudal apresentando um componente cístico em seu interior, como evidenciado na Figura 1. Sugeriu-se processo neoplásico de origem em lobo esquerdo da tireoide. O hipertireoidismo desenvolve-se a partir do tecido tireoidiano normal para um processo hiperplásico e por sua vez, adenoma tireoideano e, raramente, carcinoma (Peterson, 2020). Gatos com essa neoplasia maligna podem apresentar sinais clínicos decorrentes da elevação de T4 comumente observados no hipertireoidismo (Hibbert et al., 2009), como diarréia e taquicardia encontrados no animal do caso.

A tomografia computadorizada vem sendo reconhecida como uma das possibilidades diagnósticas do hipertireoidismo em gatos, podendo esta determinar a localização e extensão da doença com precisão e estimar uma melhor dosagem de metimazol 
Research, Society and Development, v. 10, n. 16, e304101624069, 2021

(CC BY 4.0) | ISSN 2525-3409 | DOI: http://dx.doi.org/10.33448/rsd-v10i16.24069

necessária para o tratamento dessa patologia (Lautenschlaeger et al., 2013; Bush, Nemanic, Gordon, \& Bobe, 2016).

Figura 1. Imagens tomográficas evidenciando massa localizada em região cervical ventral, com dimensões aproximadas de $2,31 \mathrm{~cm}$ de comprimento $\mathrm{x} 0,85 \mathrm{~cm}$ de largura $\mathrm{x} 0,89 \mathrm{~cm}$ de altura.

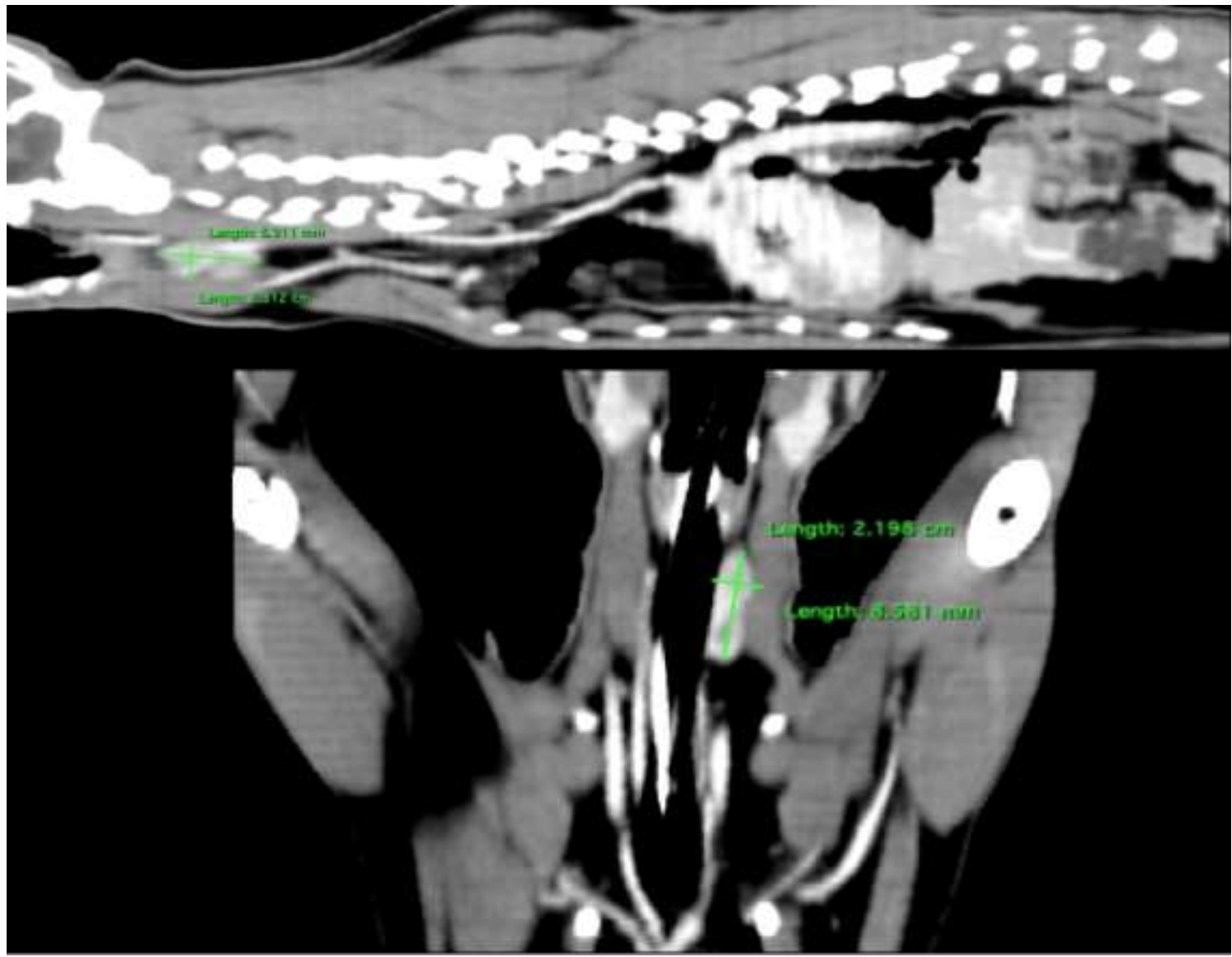

Fonte: Autores.

A mensuração de T4 foi seriada ao longo de todo tratamento e foi fundamental para o diagnóstico e acompanhamento do animal, como mostrado na Figura 2. Ao primeiro exame o T4 total estava elevado acima de seu limite superior o que é comum em animais com hipertireoidismo e que segundo Peterson (2006) é extremamente específico para o diagnóstico de hipertireoidismo. O aumento da tireóide detectado no exame físico concordou com Norsworthy et al., (2002), os quais destacam que esse achado clínico está correlacionado com o aumento de T4 em gatos com essa doença, como foi observado no presente caso. 
Figura 2: Mensuração de T4 total ao longo de todo tratamento. *: primeira tireoidectomia. **: segunda tireoidectomia.
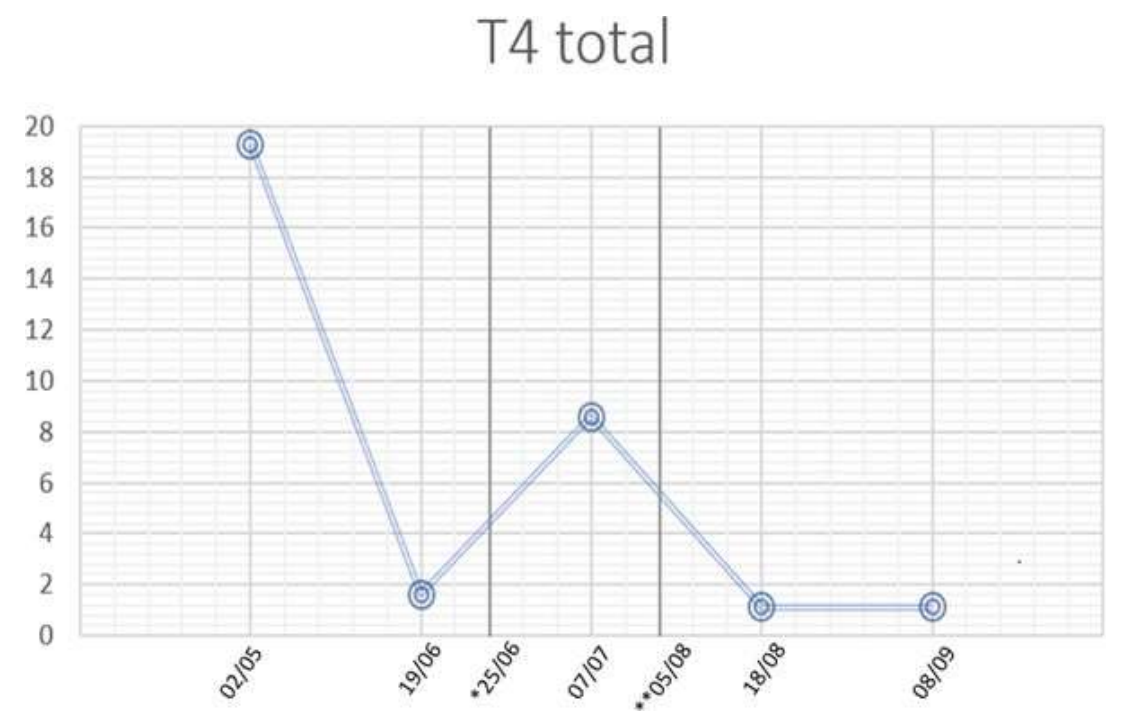

Fonte: Autores.

Após a instituição de fármaco antitireoidiano, metimazol 2,5 mg/gato BID, o animal apresentou uma diminuição considerável do T4 total. Este fármaco é instituído na fase inicial do tratamento para estabilizar o animal e reverter seus sinais clínicos, além de ser a conduta mais indicada antes da tireoidectomia (Behrend, 2006). Carney et al., (2016) afirmam que os gatos com hipertireoidismo possuem risco aumentado de complicações decorrentes de alterações cardiovasculares ou mesmo de um hipertireoidismo grave, logo, o tratamento prévio com metimazol tem indicação benéfica anterior ao tratamento definitivo, seja a tireoidectomia ou o tratamento com iodo radioativo. É importante ressaltar que o acompanhamento da função renal do paciente anterior e durante o tratamento com metimazol, pode revelar uma doença renal crônica, comum em gatos com hipertireoidismo e geriátricos (Daminet et al., 2014). Isto se deve ao fato que o hipertireoidismo aumenta débito cardíaco, diminui resistência vascular periférica e o aumento da taxa de filtração glomerular subestima o valor de creatinina circulante (Van Hoek \& Daminet, 2009). No caso em questão o animal ainda continuou sendo avaliado hematológica e bioquimicamente após o procedimento cirúrgico devido a tais considerações.

A tireoidectomia é uma opção bastante eficaz e indicada de tratamento para gatos com hipertireoidismo, pois oferece cura permanente tanto para adenomas, como carcinomas e sem a necessidade de tratamento medicamentoso ininterrupto (Birchard, 2006; Carney et al., 2016). Atualmente a técnica extracapsular modificada com abordagem cervical, que preserva as paratireóides, é indicada por ser considerada a de menor probabilidade de desenvolver quadros de hipocalcemia futuros, logo esta foi a técnica de escolha neste caso (Lavallee, Olson \& Norswhorthy, 2018; Little, 2015). Essa técnica, que deve ser realizada com bastante delicadeza, consiste na identificação da tireóide acometida e realizada cuidadosamente sua dissecção. Em seguida, a paratireóide na parte cranial é localizada e separada da tireóide por meio de cauterização prévia da cápsula da tireóide a $2 \mathrm{~mm}$ da paratireóide ou separação por dissecção com aplicadores com ponta de algodão estéril. Após a completa separação da glândula paratireóide, retira-se a tireóide acometida (North \& Banks, 2009; Borges et al., 2010). A técnica descrita por Fossum (2019) está ilustrada na Figura 3. Foi realizada remoção cirúrgica de lobo tireoidiano esquerdo e instituída prescrição de marbofloxacina $3 \mathrm{mg} / \mathrm{kg}$ SID, Meloxicam 0,05mg/kg SID além de Metimazol com frequência reduzida 2,5 mg/gato SID). 
Figura 3: Tireoidectomia extracapsular modificada destacando a remoção da tireóide, separando-a do tecido circundante e da paratireoide.

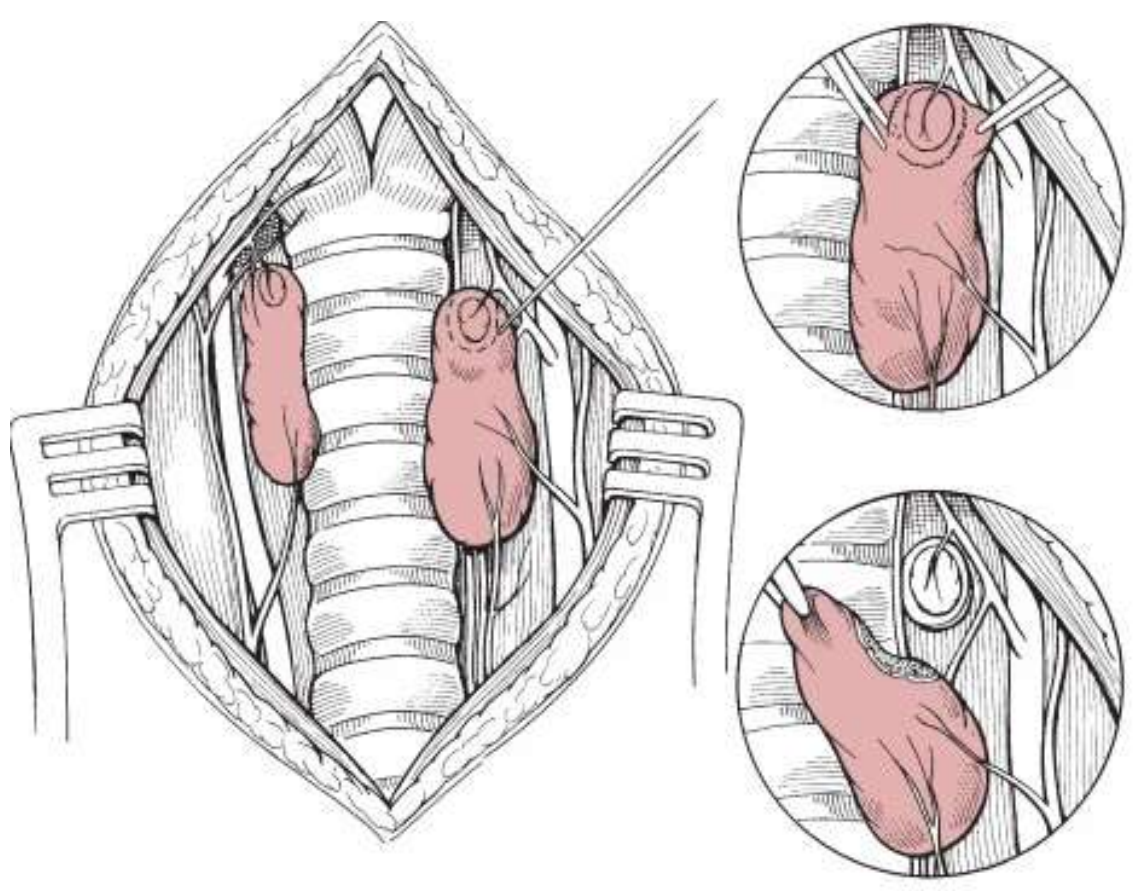

Fonte: Macphail \& Fossum (2019).

O fragmento retirado foi encaminhado para exame histopatológico, o qual concluiu que se tratava de um adenocarcinoma ductal sólido bem diferenciado. Casos de carcinoma de tireoide em felinos são bastante raros, ocorrendo em apenas 2\% dos gatos com hipertireoidismo (Lavalle, Olson \& Norsworthy, 2018). Após 20 dias da cirurgia, uma nova mensuração de T4 foi realizada e demonstrou-se ainda um valor maior que o limite normal (Figura 1), e então foi realizado novo procedimento de tireoidectomia para a retirada do lobo direito, e após esta última, os níveis de T4 foram controlados.

Como resultado da análise histopatológica do fragmento, observou-se que a arquitetura tissular está quase completamente obliterada por transformação neoplásica expansiva, infiltrativa, pouco delimitada e parcialmente revestida por cápsula fibrosa espessa, como mostra a Figura 4-B. As células neoplásicas são poliédricas, grandes e exibem núcleo grande, redondo e centralizado, citoplasma abundante eosinofílico vacuolar, como mostrado na Figura 4-A. 
Figura 4: Fotomicrografia da neoplasia (HE: 40x). Na figura A, células neoplásicas (seta preta) com presença de vacúolos (seta vermelha). Na figura B, estruturas ductais contendo colóide (seta preta); também na figura B, cápsula fibrosa espessa (seta vermelha).

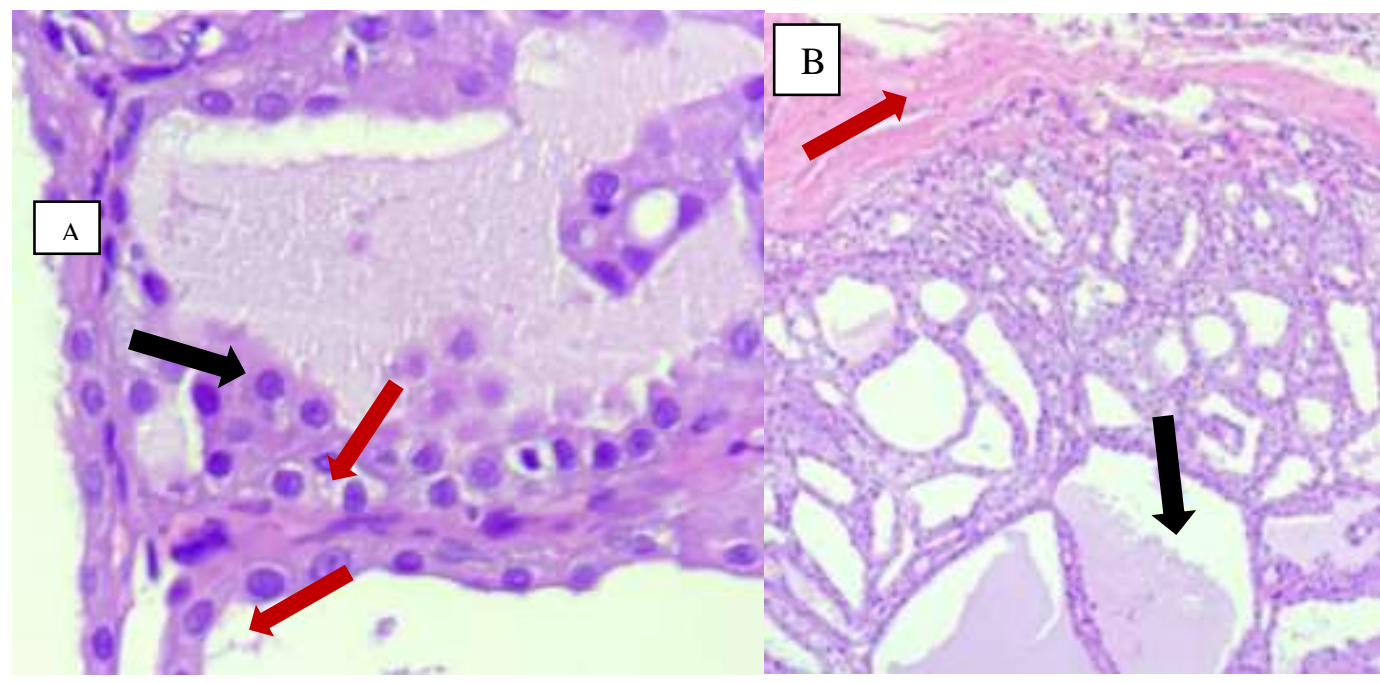

Fonte: Autores.

As mensurações de cálcio total realizadas após o segundo procedimento cirúrgico indicaram que a uma queda esperada na concentração do íon. A hipocalcemia pode desenvolver quadros de letargia, anorexia, fasciculações, resistência ao se movimentar, tetania e convulsões. A suplementação de cálcio é indicada nos casos em que a sua concentração sérica está menor que $7 \mathrm{mg} / \mathrm{dL}$ (Brichard, 2006). Neste caso, a concentração se manteve em torno de $6,5 \mathrm{mg} / \mathrm{dL}$ nas cinco avaliações realizadas após a segunda tireoidectomia. Assim, foi instituída suplementação de cálcio (Cal-D-Mix® 1⁄2 comprimido SID) por 40 dias. Trinta dias após o início da suplementação os valores de cálcio sérico foram normalizados (8,6mg/dL).

\section{Conclusão}

O hipertireoidismo ocasionado por carcinoma é uma enfermidade incomum na clínica de felinos, dessa forma é necessário que o médico veterinário relacione os sinais clínicos e o exame físico, para que solicite os exames necessários, se obtenha um diagnóstico definitivo e para que adote uma terapêutica adequada.

\section{Referências}

Behrend, E. N. (2006). Update on drugs used to treat endocrine diseases in small animals. Veterinary Clinics: Small Animal Practice, 36(5), 1087-1105.

Birchard, S. J. (2006). Thyroidectomy in the cat. Clinical Techniques in Small Animal Practice, 21(1), 29-33.

Borges, D. S., Casarin, C. S., Borges, L. T., \& Leite, A. T. M. (2010). Tireoidectomia extracapsular com transplante de paratireóide em felino-Relato de caso. MEDVEP. Revista Científica de Medicina Veterinária, 593-597.

Bush, J. L., Nemanic, S., Gordon, J., \& Bobe, G. (2016). Computed tomographic characteristics of the thyroid glands in eight hyperthyroid cats pre- and post methimazole treatment compared with seven euthyroid cats. Veterinary Radiology \& Ultrasound, 1-10.

Daleck, C. R., \& De Nardi, A. B. (2016). Oncologia em cães e gatos . Grupo Gen-Editora Roca Ltda.

Daminet, S., Kooistra, H. S., Fracassi, F., Graham, P. A., Hibbert, A., Lloret, A., Mooney, C. T., Neiger, R., Rosenberg D., Villard, I. \& Williams, G. (2014). Best practice for the pharmacological management of hyperthyroid cats with antithyroid drugs. Journal of Small Animal Practice, 55(1), 4-13.

Gunn-Moore, D. (2005). Feline endocrinopathies. Veterinary Clinics: Small Animal Practice, 35(1), 171-210.

Hibbert, A., Gruffydd-Jones, T., Barrett, E. L., Day, M. J., \& Harvey, A. M. (2009). Feline thyroid carcinoma: diagnosis and response to high-dose radioactive iodine treatment. Journal of Feline Medicine and Surgery, 11, 116-124.

Lautenschlaeger, I. E., Hartmann, A., Sicken, J., Mohrs, S., Scholz, V. B., Neiger, R., \& Kramer, M. (2013). Comparison between computed tomography and 
Research, Society and Development, v. 10, n. 16, e304101624069, 2021

(CC BY 4.0) | ISSN 2525-3409 | DOI: http://dx.doi.org/10.33448/rsd-v10i16.24069

99m TC- pertechnetate scintigraphy characteristics of the thyroid gland in cats with hyperthyroidism. Veterinary Radiology \& Ultrasound, 54, 666-673.

Lavallee, J., Olson, J. K., \& Norsworthy, G. D. (2018). HypertireodismIn The feline patient (5a ed). Wiley Blackwell.

Little, S. E. (2015). O gato: medicina interna; tradução Roxane Gomes dos Santos Jacobson, Idilia Vanzellotti.

Macphail, C., \& Fossum, T. W. (2019). Surgery of the endocrine system. In: Fossum, T. W. (Org.), Small Animal Surgery (5a ed) (Cap. 22, pp. 586-630). USA: Mosby, inc.

Mooney, C. T. (2001). Feline hyperthyroidism. Veterinary Clinics of North America: Small Animal Practice, 31(5), 963-983.

Norsworthy, G. D., Adams, V. J., McElhaney, M. R., \& Milios, J. A. (2002). Relationship between semi-quantitative thyroid palpation and total thyroxine concentration in cats with and without hyperthyroidism. Journal of feline medicine and surgery, 4(3), 139-143.

North, S., \& Banks, T. (2009). Introduction to small animal oncology. Saunders. California, USA, 159-160.

Peterson, M. (2012). Hyperthyroidism in cats: what's causing this epidemic of thyroid disease and can we prevent it?. Journal of feline medicine and surgery, 14(11), 804-818.

Peterson, M. E. (2006). Diagnostic tests for hyperthyroidism in cats. Clinical techniques in small animal practice, $21(1), 2-9$.

Peterson, M. E. (2020). Hyperthyroidism in cats: considering the impact of treatment modality on quality of life for cats and their owners. Veterinary Clinics: Small Animal Practice, 50(5), 1065-1084.

Vail, D. M., Thamm, D. H., \& Liptak, J. M. (2019). Hematopoietic tumors. Withrow and MacEwen's small animal clinical oncology, 688.

Van, Hoek, I., \& Daminet, S. (2009). Interactions between thyroid and kidney function in pathological conditions of these organ systems: a review. General and comparative endocrinology, 160(3), 205-215. 